\title{
DESIGN AND SIMULATION OF PARALLEL CDMA SYSTEM BASED ON 3D-HADAMARD TRANSFORM
}

\author{
Ali T. Shaheen \\ Assistant Lecturer, Electrical Eng. Dept., College of Engineering, University of Baghdad \\ E-mail:alitweshaheen@yahoo.com \\ (Received: 29/4/2012; Accepted:26/11/2012)
}

\begin{abstract}
Future wireless systems aim to provide higher transmission data rates, improved spectral efficiency and greater capacity. In this paper a spectral efficient two dimensional (2-D) parallel code division multiple access (CDMA) system is proposed for generating and transmitting (2-D CDMA) symbols through 2-D Inter-Symbol Interference (ISI) channel to increase the transmission speed. The 3D-Hadamard matrix is used to generate the 2-D spreading codes required to spread the two-dimensional data for each user row wise and column wise. The quadrature amplitude modulation (QAM) is used as a data mapping technique due to the increased spectral efficiency offered. The new structure simulated using MATLAB and a comparison of performance for serial one-dimensional (1D) CDMA and parallel (2-D) CDMA is made under Additive White Gaussian Noise (AWGN), flat fading and multi-path selective fading channels conditions. It is found that 2-D CDMA has better speed and performance than serial 1-D CDMA.
\end{abstract}

Keywords: DS-CDMA, 3-D Hadamard, 2-D Spreading Codes, 2-D CDMA, 2-D ISI Channel

\section{1- INTRODUCTION}

The demand for higher data rates and higher quality in wireless communication systems has recently seen unprecedented growth. One of the most limiting factors in wireless communications is the scarcity of spectrum. Techniques that improve spectral efficiency, such as the cellular structure that allows frequency reuse, have had tremendous impact on the proliferation of wireless communications ${ }^{(1)}$. The next generation of wireless systems will require higher data quality than current cellular mobile radio systems and should provide higher bit rate services. In other words, the next generation of wireless systems are supposed 
to have a better quality and coverage, be more powerful and bandwidth efficient, and be deployed in diverse environments (2). The ever-increasing demand for wireless and multimedia applications such as video streaming keeps pushing future wireless LAN (WLAN) systems to support much higher data rates (100 MB/s up to $1 \mathrm{~GB} / \mathrm{s})$ at high link reliability and over greater distances. Next-generation wireless communication systems are focused on increasing the link throughput (bit rate), the network capacity, and the transmit range. This can be achieved at the cost of increased signal processing complexity. The required algorithms are usually derived analytically from mathematical models based on many simplifying assumptions. Following this, the original, "optimal" procedures nearly always need to be modified (i.e., reengineered) in order to reduce their computational complexity ${ }^{(3,4)}$. A two - dimensional OFDM system is proposed in [5] as spectral efficient OFDM system, to generate and transmit a two dimensional OFDM symbols through (2-D ISI) channel to increase the transmission speed with a Bit Error Rate (BER) performance improvement. In this paper a two dimensional (2-D) parallel code division multiple access (CDMA) system is proposed to increase the transmission speed by generating and transmitting (2-D CDMA) symbols through ( 2-D ISI) channel. The 3-D Hadamard transform is used as a main building block in the proposed parallel CDMA system which used to generate the two dimensional spreading codes that required to spreads the 2-D data of each user row wise and column wise.

\section{2-Three-Dimensional Hadamard Transform}

Walsh code sequences that used in the conventional CDMA systems are obtained from the Hadamard matrix which is a square matrix where each row in the matrix is orthogonal to all other rows, and each column in the matrix is orthogonal to all other columns. The Hadamard matrix $\mathrm{H}_{\mathrm{n}}$ is $N \times N$ matrix, where $N=2^{n}$. These can be generated by the core matrix

$$
H_{1}=\frac{1}{\sqrt{2}}\left[\begin{array}{cc}
1 & 1 \\
1 & -1
\end{array}\right]
$$

And applying the Hadamard transform successively using the Kronecker product recursion [6-9]

$$
\begin{aligned}
& H_{n}=H_{n-1} \otimes H_{1} \\
& H_{n}=\frac{1}{\sqrt{2^{n}}}\left[\begin{array}{cc}
H_{n-1} & H_{n-1} \\
H_{n-1} & -H_{n-1}
\end{array}\right]
\end{aligned}
$$


As an example, for $n=3$, the Hadamard matrix becomes

\section{Sequency}

$$
H_{3}=\frac{1}{\sqrt{8}}\left[\begin{array}{rrrrrrrr|l}
1 & 1 & 1 & 1 & 1 & 1 & 1 & 1 & 0 \\
1 & -1 & 1 & -1 & 1 & -1 & 1 & -1 & 7 \\
1 & 1 & -1 & -1 & 1 & 1 & -1 & -1 & 3 \\
1 & -1 & -1 & 1 & 1 & -1 & -1 & 1 & 4 \\
1 & 1 & 1 & 1 & -1 & -1 & -1 & -1 & 1 \\
1 & -1 & 1 & -1 & -1 & 1 & -1 & 1 & 6 \\
1 & 1 & -1 & -1 & -1 & -1 & 1 & 1 & 2 \\
1 & -1 & -1 & 1 & -1 & 1 & 1 & -1
\end{array}\right]
$$

Each column or row in the Hadamard matrix corresponds to a Walsh code sequence of length $n$. Orthogonality between codes in the Hadamard matrix is defined such that the crosscorrelation values, associated with zero offset between the pair of sequences is zero ${ }^{(6)}$.

The number of zero crossings of a Walsh function or the number of transitions in a basis vector of the Hadamard transform is called its sequency (as well as the sinusoidal signals frequency can be defined in terms of the zero crossings).

In the Hadamard matrix generated through eq. (3), the row vectors are not sequency ordered, as shown in eq. (4) by the column named "Sequency". The existing sequency order of these vectors is called the Hadamard order because this is the order used by the transform ${ }^{(9)}$. In conventional CDMA system each sequence is assigned to one user thus, an NxN Walsh matrix can be used to multiplex $\mathrm{N}$ users in CDMA system.

The 3-D Hadamard matrix can be simply generated by the Kronecker product of the 3-D Hadamard core matrix $[9,10]$.

Figure (1) show the generation of 3D Hadamard matrix.

As shown in Figure 1 the 3D-Hadamard core matrix is:

$$
\mathrm{H}_{2 \times 2 \times 2}=\begin{array}{rr}
-1 & -1 \\
-1 & 1 \\
1 & 1 \\
1 & -1 \\
\hline
\end{array}
$$

The $(\mathrm{N} \times \mathrm{N} \times \mathrm{N})$ 3-D Hadamard matrix can be generated in similar manner as in the 2-D Hadamard matrix by the Kronecker product of the 3-D Hadamard core matrix.

$$
H_{\mathrm{N} \times \mathrm{N} \times \mathrm{N}}=H_{(\mathrm{N}-1)(\mathrm{N}-1)(\mathrm{N}-1)} \otimes H_{2 \times 2 \times 2}
$$


Thus

$\mathrm{H}_{4 \times 4 \times 4}=\quad$\begin{tabular}{cc|}
$-\mathrm{H}_{2 \times 2 \times 2}$ & $-\mathrm{H}_{2 \times 2 \times 2}$ \\
$-\mathrm{H}_{2 \times 2 \times 2}$ & $\mathrm{H}_{2 \times 2 \times 2}$ \\
$\mathrm{H}_{2 \times 2 \times 2}$ & $\mathrm{H}_{2 \times 2 \times 2}$ \\
$\mathrm{H}_{2 \times 2 \times 2}$ & $-\mathrm{H}_{2 \times 2 \times 2}$ \\
\hline
\end{tabular}

The $(\mathrm{N} \times \mathrm{N} \times \mathrm{N})$ 3D-Hadamard matrix, consists of two dimensional matrices $\mathrm{C}(:,:, \mathrm{j})$ of dimension $(\mathrm{N} \times \mathrm{N})$ where $\mathrm{j}=1,2, \ldots, \mathrm{N}$; each one of these matrices will be orthogonal with all other matrices:

$\sum_{j=0}^{N-1} C(:,:, i) \cdot * C(:,:, i+j)=0$

Where $i=1,2 \ldots N / 2$

In this paper we use each one of these two dimensional matrices as a two dimensional spreading code in the proposed parallel CDMA system.

\section{Two-Dimensional Channel Model}

Two-Dimensional finite state input channels with memory plays a fundamental role in various applications in modern communications. A popular and important instance of this class of channels is 2-D ISI channels, which appear, e.g. in magnetic and optical recording systems ${ }^{(11)}$. In ISI finite state symbols are ordered on a 2-D grid, causing interference in a limited neighborhood. Another interesting instance of dispersive 2-D channels is multipleaccess (MA) channels, which appear in cellular networks. Following Wyner's cellular model (12), a planer uplink model can be viewed as a 2-D channel, where each cell corresponds to a node in the grid, and interference occurs between neighboring cells. This Wyner-like model assumes that most of the multiple- access interference (MAI) is caused by inter-cell effects, rather than intra-cell effects.

Many researches have proposed 2-D ISI channel detection schemes model, [13, 11]. Singla et al. have proposed joint equalization and decoding schemes for 2-D ISI discrete channels, and this is the channel model used in this paper for simulation of the proposed 2-D CDMA system. This channel model is binary-input, linear finite-state ISI channel where the input to this channel is 2-D matrix of size $\left(L_{1}, L_{2}\right)$. The output of this channel is described by 
$\boldsymbol{r}_{(i, j)}=\sum_{k 1, k 2=0}^{L 1, L 2} \boldsymbol{x}_{(i-k 1, j-k 2)} \boldsymbol{h}_{\left(k_{1}, k_{2}\right)} \boldsymbol{W}_{(i, j)}$

where $r_{(i, j)}$ and $x_{(i, j)}$ are elements of output and input data matrices respectively; $w_{(i, j)}$ are samples of additive white Gaussian noise (AWGN) with variance $\sigma^{2} ; h$ is the 2-D channel impulse response:

$h=\left[\begin{array}{ll}h_{0} & h_{1} \\ h_{2} & h_{3}\end{array}\right]$

For example, an impulse response used in simulation in [5] is

$h=\left[\begin{array}{cc}1 & 0.5 \\ 0.5 & 0.2\end{array}\right]$

Hint: the sign (.*) refers to element by element multiplication.

This channel response is separable into

$h=\left[\begin{array}{ll}1 & 0.5\end{array}\right]^{T}\left[\begin{array}{ll}1 & 0.5\end{array}\right]$.

In eq. (9) $L_{1}, L_{2}$ represents number of elements over which ISI extends in each dimension. The channel impulse response can be divided in to two types: non separable ISI cannel and separable ISI channel as shown in Figure (2).

\section{The Proposed Two-Dimensional CDMA System}

The conventional serial CDMA system transmit data serially, whereas the proposed 2D parallel CDMA system transmits data in parallel (the transmitted signal is 2-D CDMA signal). This increases transmission speed, since parallel transmission is much faster than serial transmission and so this system is suitable for applications requiring high data rates.

The proposed Two-Dimensional CDMA system is shown in Figure (3). This system generates and transmits a two-dimensional CDMA symbols through a two-dimensional Inter Symbol Interference Channel (2-D ISI Channel) to increase the transmission speed by allowing the system to transmit 2-D data for each user instead of 1-D data as in the conventional system. The proposed system is simulated using MATLAB and described in the flow chart shown in Figure (4).

The data of each user must be two-dimensional data, and the system use two dimensional spreading codes (to spread the user 2-D data row wise and column wise) generated by using the 3-D Hadamard matrix described above. $(N \times N \times N)$ 3-D Hadamard matrix generates $(N)$ two dimensional orthogonal slides of size $(N \times N)$; each one can be used to spread the 2-D data of one user i.e. we have $N$ users CDMA system as shown in Figure (5). 
Let us consider that the data of each user is 2-D data of size $\left(M_{1}, M_{2}\right)$ described as $d_{i}\left(k_{1}, k_{2}\right) \quad$ where $i \leq N$ and $N>\max \left(M_{1}, M_{2}\right)$

Then the 2-D CDMA signal is

$S\left(t_{1}, t_{2}\right)=\sum_{i=1}^{N} d i(k 1, k 2) \otimes C i(l 1, l 2)$

where $C_{i}\left(l_{1}, l_{2}\right)$ is the two-dimensional spreading code that used for the $i_{t h}$ user.

The processing gain for this system will depend on the value of $N$. The final baseband data $S$ will be of size $\left(N \times M_{1}, N \times M_{2}\right)$, this two-dimensional data can be transmitted through a twodimensional channel. By this method the data rate (and then the transmission speed) will be increased significantly as compared with the conventional system.

\section{Case Study}

Figure (6). Shows an illustrative numerical example for a 2-user parallel 2-D CDMA system. Consider $(4 \times 4 \times 4) 3$-D Hadamard matrix given in Figure (1) (b) this matrix gives four spreading codes each one of dimension $(4 \times 4)$.

As given in Figure 5; the data of each user must be two-dimensional data.

Let us consider the dimension of these data is $2 \times 2$.

$\mathrm{D}_{1}=\left[\begin{array}{rr}1 & 1 \\ -1 & -1\end{array}\right] \quad ; \mathrm{D}_{2}=\left[\begin{array}{ll}1 & 1 \\ 1 & 1\end{array}\right]$

The baseband 2-D CDMA symbol will results from the summation of the two matrices that results from the Kronecker product between the user data and the 2-D spreading code. As given in Figure 6 the dimension of the result will be $(8 \times 8)$.

At the receiver side the process used to recover the data of each user is similar to that of the conventional system but in two dimensional fashion. The data of the $1^{\text {st }}$ user will be recovered by using the following equation

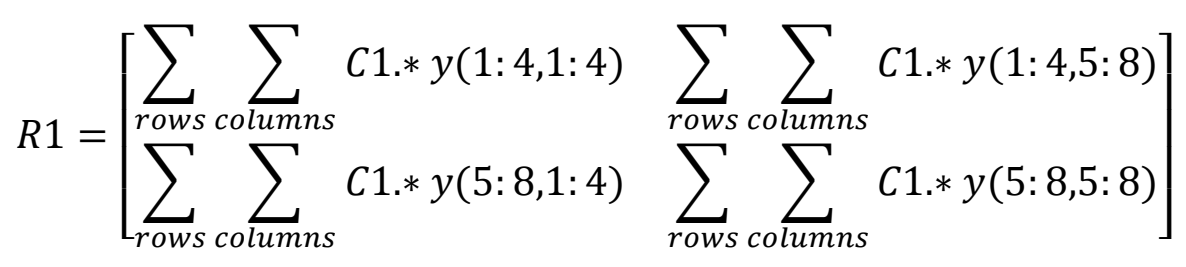

Where $\mathbf{y}$ is the two dimensional CDMA symbol as given in Figure (6). Thus R1 will be:

$\mathrm{R}_{1}=\left[\begin{array}{cc}16 & 16 \\ -16 & -16\end{array}\right]$

If we replace $C_{1}$ in eq.(14) by $C_{2}$ then the result will be

$\mathrm{R}_{2}=\left[\begin{array}{ll}16 & 16 \\ 16 & 16\end{array}\right]$ 
By dividing R1 and R2 by 16 , the data of user $_{1}$ and user $_{2}$ are recovered

\section{Simulation and BER performance of the proposed 2-D parallel CDMA system.}

The proposed 2-D CDMA system described in the previous section is simulated for various numbers of active users and its BER performance is compared with the conventional standard 1-D CDMA system. In this simulation a $(16 \times 16 \times 16) 3-D$ Hadamard matrix is used to generate 16 two -dimensional spreading codes each one of dimension equal to $(16 \times 16)$ with spreading factor equal to 16 (row wise and column wise). The mapper used in the system is 4QAM mapper, to increase the spectral efficiency of the system.

The channel models used in the simulation of the 2-D parallel CDMA are AWGN, flat fading and multi-path Raleigh distributed fading channel with impulse response equal to

$h=\left[\begin{array}{cc}1 & 0.5 \\ 0.5 & 0.25\end{array}\right] \quad$ Or; in separable form $h=\left[\begin{array}{ll}1 & 0.5\end{array}\right]^{T}\left[\begin{array}{ll}1 & 0.5\end{array}\right]$.

Figure (7) shows BER performance of the proposed 2-D CDMA system compared with BER performance of the standard 1-D CDMA system in an AWGN channel. It can be shown that the proposed system has a gain in SNR about $2.5 \mathrm{~dB}$ to achieve a BER performance of $\left(10^{-4}\right)$ as compared with the standard 1-D CDMA.

The BER performance of the 2-D and 1-D CDMA systems in flat fading channel is shown in Figure 8 , this figure shows that the proposed system performs better than the conventional CDMA system, where the proposed system has a $3.5 \mathrm{~dB}$ gain in SNR to achieve BER about $\left(10^{-4}\right)$ as compared with the conventional system.

Figure (9) shows the BER performance of the two systems in selective fading channel with second path delay of 2 samples and gain equal to $-6.3 \mathrm{~dB}$. Simulations show that 2-D parallel CDMA has better performance and it has a gain improvement in SNR about $4 \mathrm{~dB}$ to achieve a BER performance of $\left(10^{-4}\right)$ as compared with the 1-D CDMA system.

on the other hand the 2-D system has increased the transmitted data of each user by a factor of 8 since each user has 64 symbol arranged in $(8 \times 8)$ two-dimensional matrix spread by using (16×16) 2-D Walsh spreading code, where as in the standard system the data of each user is composed of 8 symbol vector spread by 16 chip Walsh code.

\section{Conclusions}


In this paper a 2-D parallel CDMA system based on two-dimensional spreading codes is proposed. This system generates 2-D CDMA symbols and transmits them through 2-D ISI channel. By this method transmission speed is increased significantly, and therefore this system is reliable for high data rate applications. Simulation results of proposed system show a good SNR gain improvement compared with the conventional 1-D CDMA system in AWGN, flat fading, and a selective fading channels. Thus this system increases the bit rate of each user by allowing each user to transmit a 2-D data with BER performance improvement due to the row wise and column wise spreading. In the designed system a (16×16×16) 3-D Hadamard matrix is used to generate 16 two -dimensional spreading codes each one of dimension equal $(16 \times 16)$ the bit rate increased by a factor of 8 with some increasing in the cost of signal processing complexity.

\section{References}

1. Da-shan Shiu, "Wireless Communication Using Dual Antenna Arrays" Kluwer Academic Publishers, 2002.

2. S.M. Alamouti, "A simple transmit diversity technique for wireless communications", IEEE J. Select Area Comm., 16, 1451-1458, 1998.

3. D.G. Rahn, M.S. Cavin, M. Toner, "A fully integrated multiband MIMO WLAN transceiver RFIC”, IEEE J. Solid-State Circuits 40, 1629-1641, (2005).

4. M. Guillaud, A. Burg, L. Mailaender, B. Haller, M. Rupp, and E. Beck, "From Basic Concept to Real-Time Implementation: Prototyping WCDMA Downlink Receiver Algorithms - A Case Study", 34th Asilomar Conf. on Signals, Systems, and Computers, Oct. 29 - Nov. 1- 2000.

5. A. H. Kattoush, W.Al-Jawhar, S. M. Abbas and Ali T. Shaheen, "A high performance parallel Radon based OFDM transceiver design and simulation”, Digital signal processing, Elsevier, USA, Vol. 18 No. 6, (907-918),November 2008.

6. M. Ali Abu-Rgheff, "Introduction to CDMA Wireless Communications", Elsevier Ltd. 2007

7. H. Hwa Chen, “The Next Generation CDMA Technologies”, John Wiley \& Sons Ltd, 2007.

8. Saad Nihad Adbul-Majeed"Multi-Carrier CDMA using multiwavelet transform" Thesis, Electrical Eng. Dept., College of Eng.,Baghdad University,2006. 
9. Vanessa Testoni and Max H. M. Costa, "3D-Hadamard Coefficients Sequency Scan Order for a Fast Embedded Color Video Codec", the 1st International Conference on Signal Processing and Communication Systems, Gold Coast, Australia, December 2007.

10. Sarah Spence Adams, Matthew Crawford, "Multilevel and multidimensional Hadamard matrices”, Springer Science+Business Media, December 2008.

11. P.S. Kumar, S. Roy, "Two-dimensional equalization: Theory and application to high density magnetic recording”, IEEE Trans. Commun. 42, 386-395,(1994).

12. A.D. Wyner, "Shanon-theoretic approach to Gaussian cellular multiple-access channel”, IEEE Trans. Inform. Theory 40, 1713-1727,(1994).

13. N. Singla, J.A. O'sullivan, R.S.Indeck, "Iterative decoding and equalization for 2-D recording channels", IEEE Trans. Magn. 38, 2328-2330,(2002).

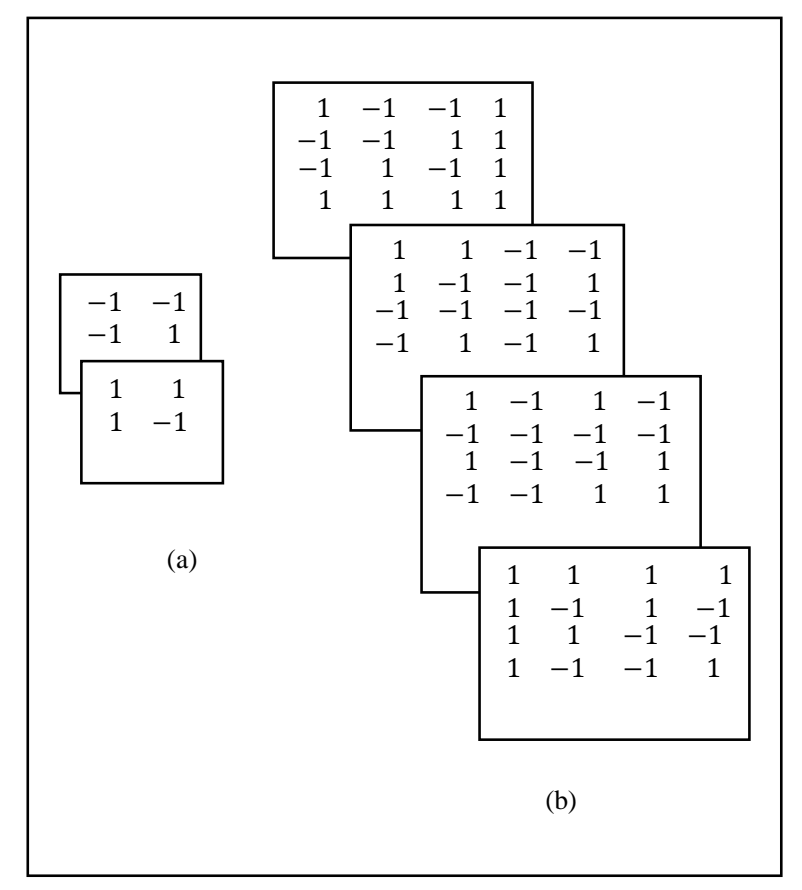

Figure (1): Three dimensional Hadamard matrix: (a) the core matrix (b): $(4 \times 4 \times 4$ Hadamard matrix) 


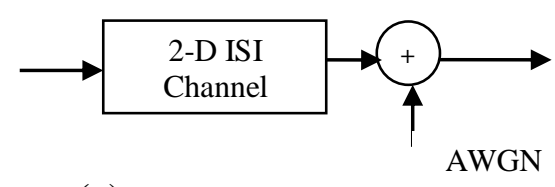

(a)

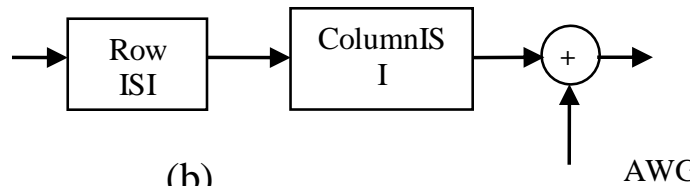

Figure (2): Two-dimensional ISI channel: (a) Non separable channel (b) separable channel

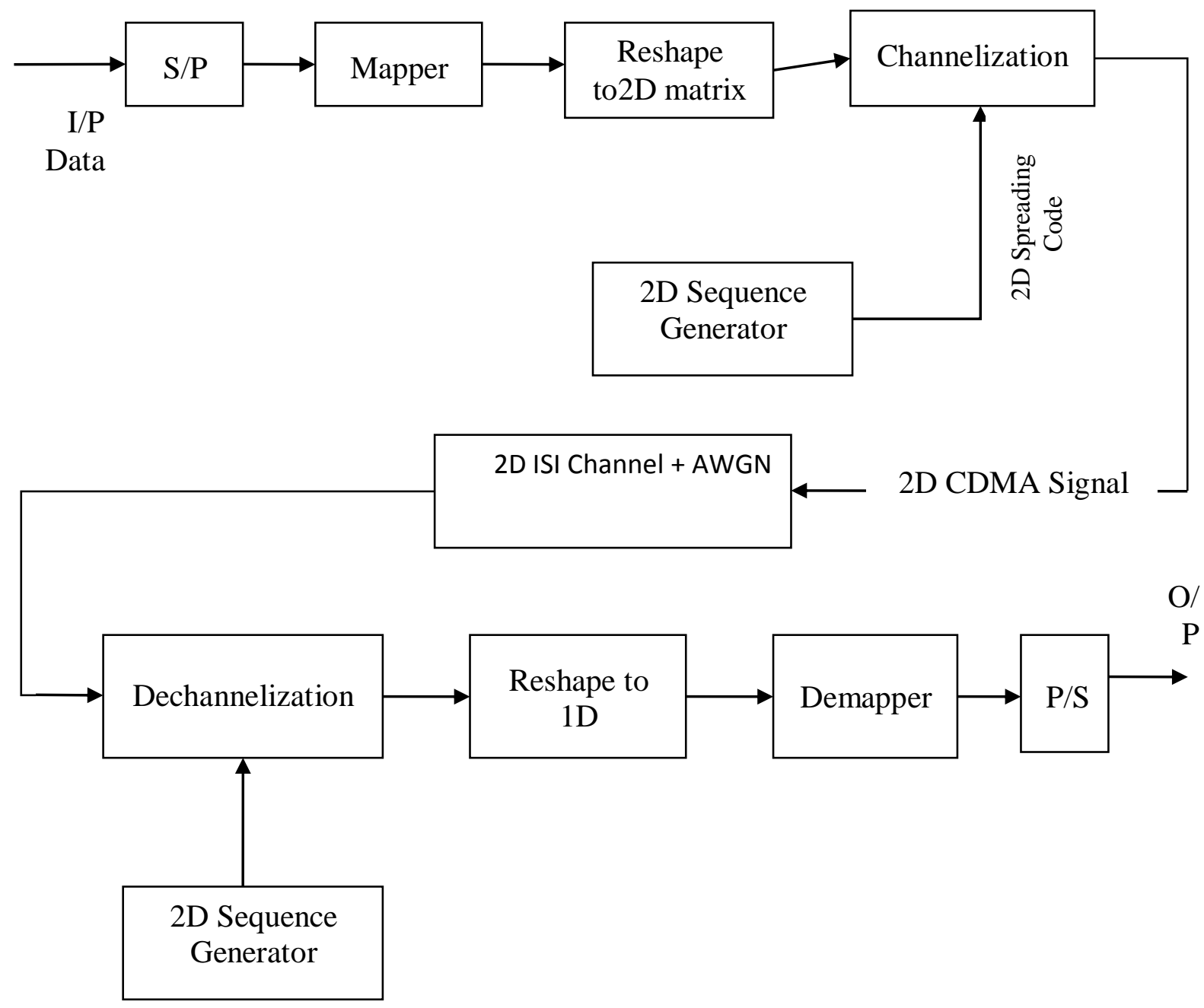

Figure 3: The proposed Two-Dimensional CDMA system. 


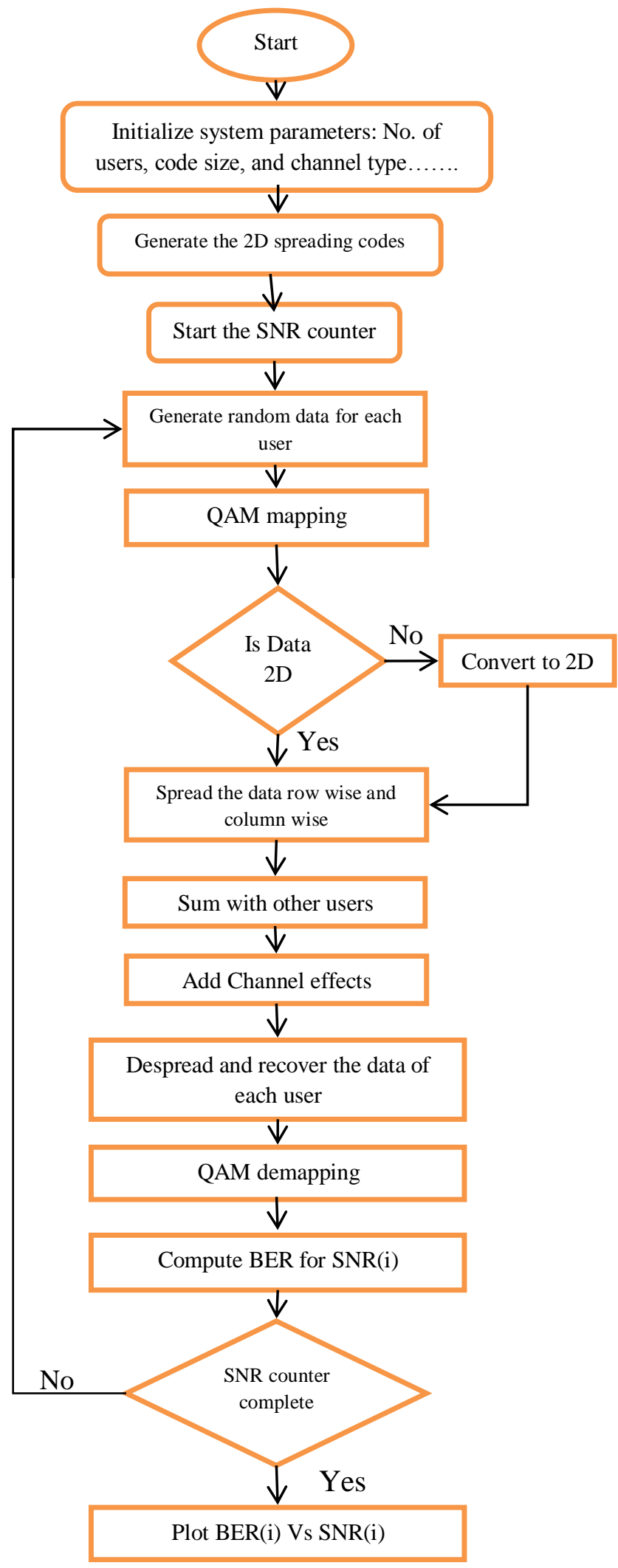

Figure (4): Flow chart for the proposed system simulation. 


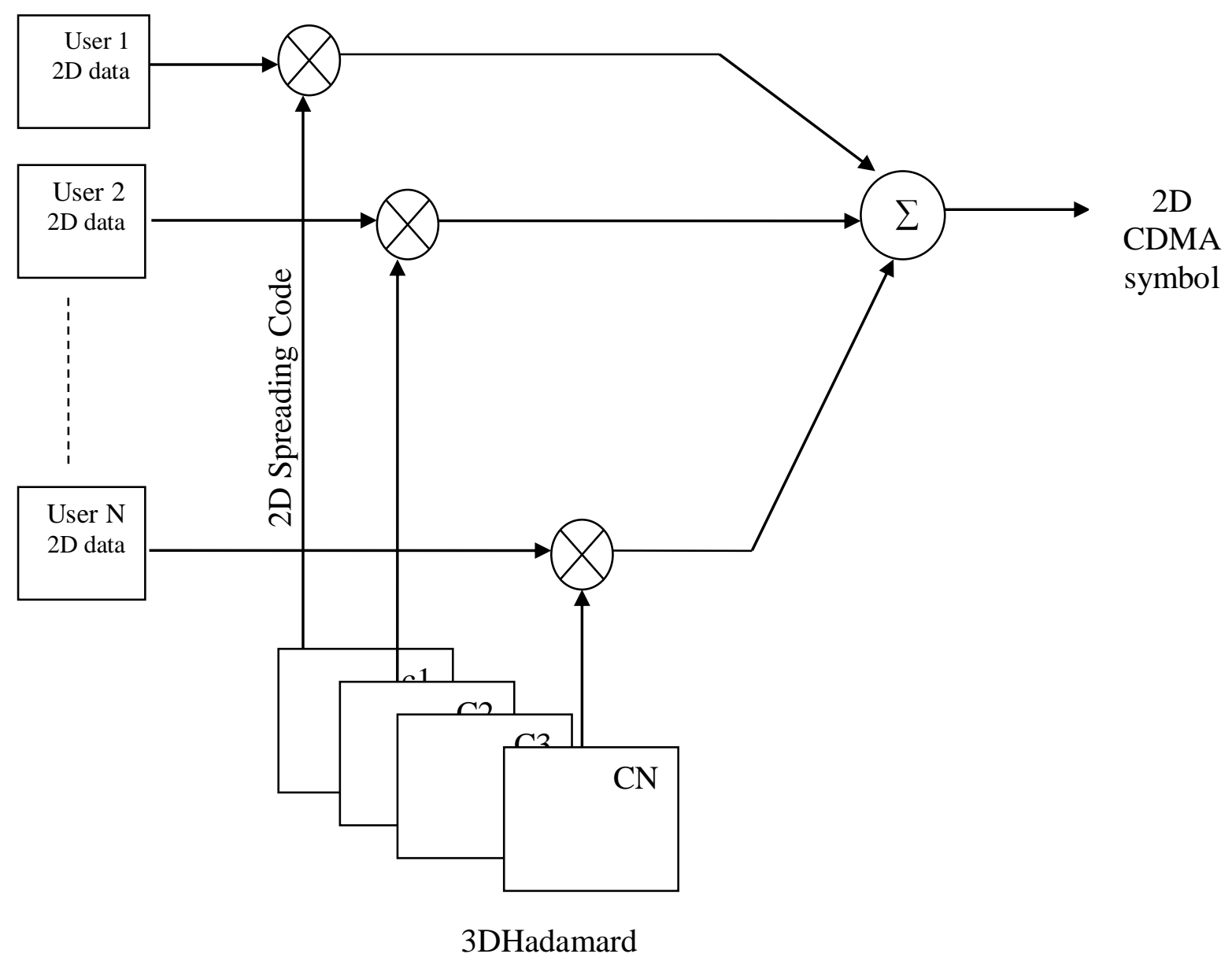

Figure (5): N- Users Two-Dimensional Transmitter based on 2-D Hadamard spreading codes. 


\begin{tabular}{|c|c|c|c|c|c|c|c|}
\hline C1 & $\begin{array}{rr}1 & 1 \\
1 & -1 \\
1 & 1 \\
1 & -1\end{array}$ & $\begin{array}{rc}1 & 1 \\
1 & -1 \\
-1 & -1 \\
-1 & 1\end{array}$ & \begin{tabular}{l|l}
1 & \\
1 & \\
&
\end{tabular} & $\begin{array}{r}1 \\
-1 \\
1 \\
-1\end{array}$ & $\begin{array}{l}-1 \\
-1 \\
-1 \\
-1\end{array}$ & $\begin{array}{r}1 \\
-1 \\
-1 \\
1\end{array}$ & $\begin{array}{r}-1 \\
-1 \\
1 \\
1\end{array}$ \\
\hline C3 & $\begin{array}{rr}1 & 1 \\
1 & -1 \\
-1 & -1 \\
-1 & 1\end{array}$ & $\begin{array}{rr}-1 & -1 \\
-1 & 1 \\
-1 & -1 \\
-1 & 1\end{array}$ & C4 & $\begin{array}{r}1 \\
-1 \\
-1 \\
1\end{array}$ & $\begin{array}{r}-1 \\
-1 \\
1 \\
1\end{array}$ & $\begin{array}{r}-1 \\
1 \\
-1 \\
1\end{array}$ & $\begin{array}{l}1 \\
1 \\
1 \\
1\end{array}$ \\
\hline
\end{tabular}

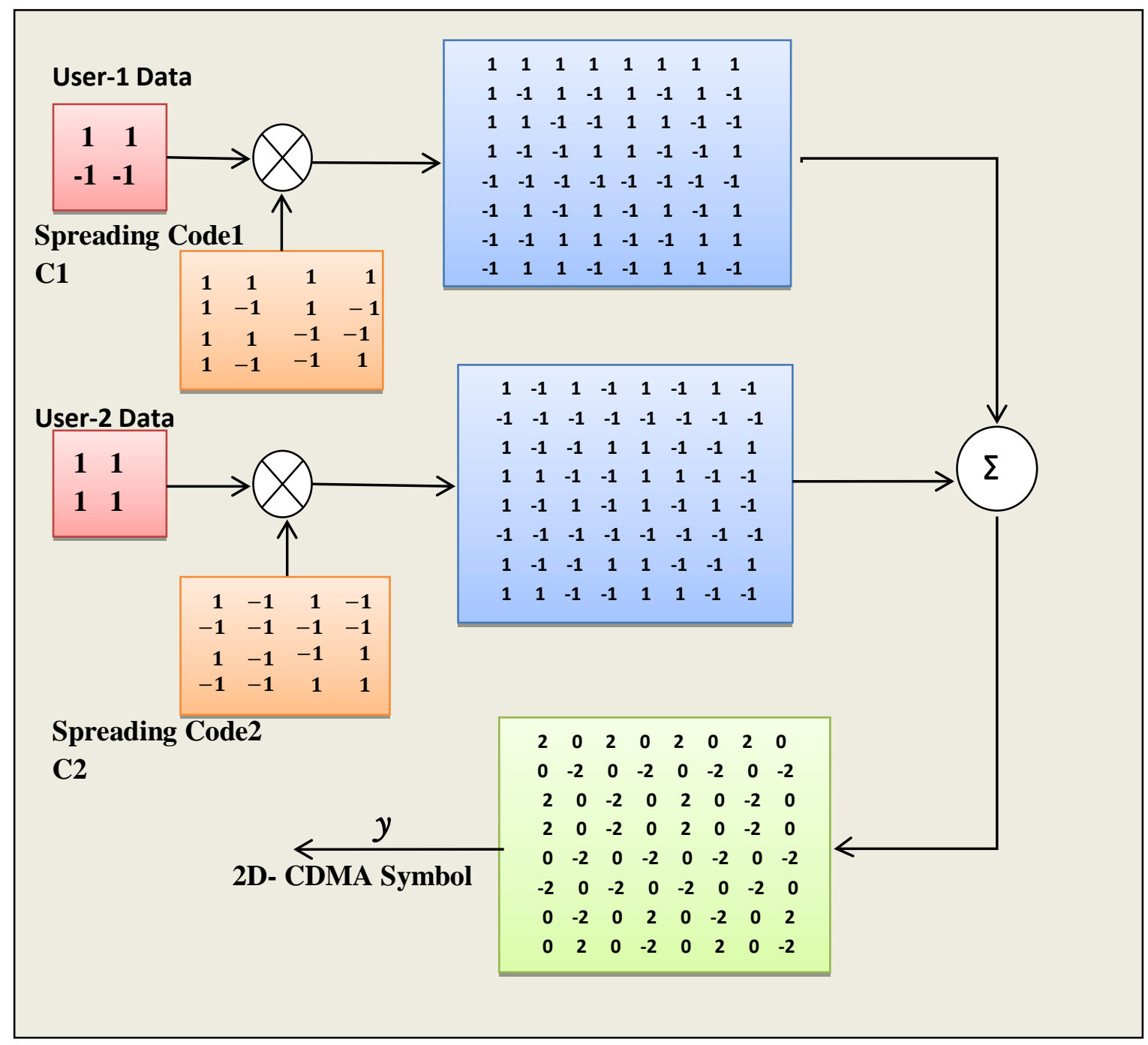

Figure (6): An illustrative numerical example of parallel 2-D CDMA 


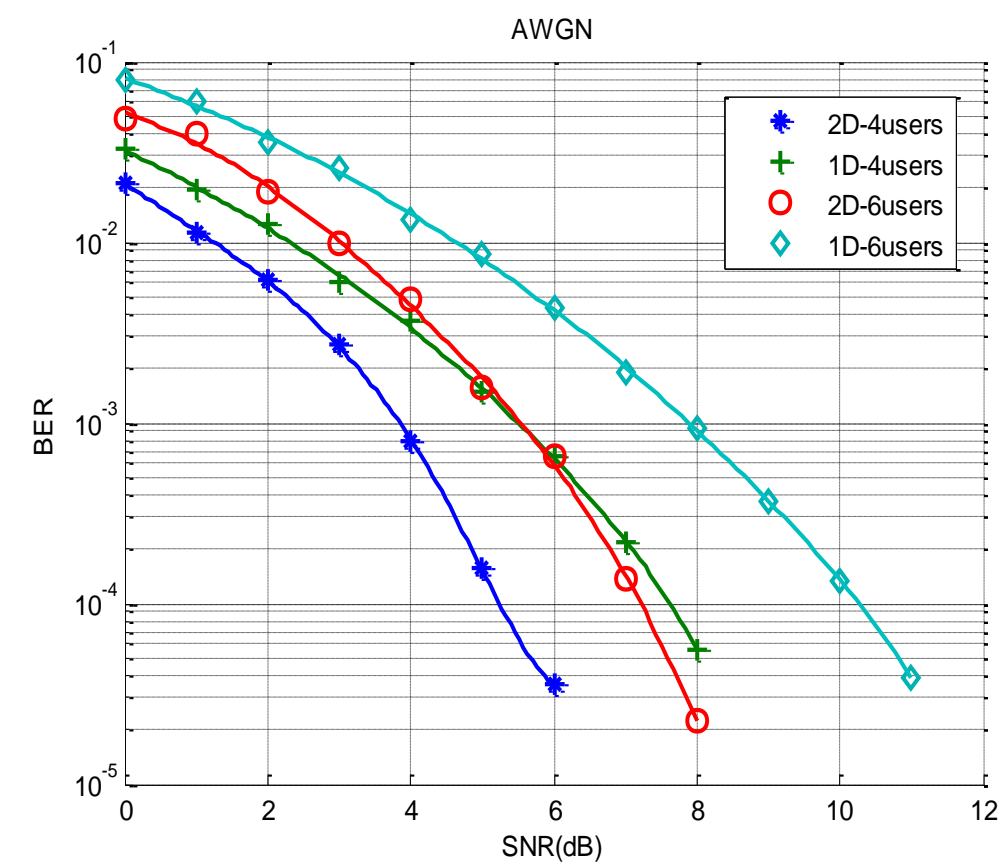

Figure (7): The performance of 2-D CDMA and 1-D CDMA in AWGN channel

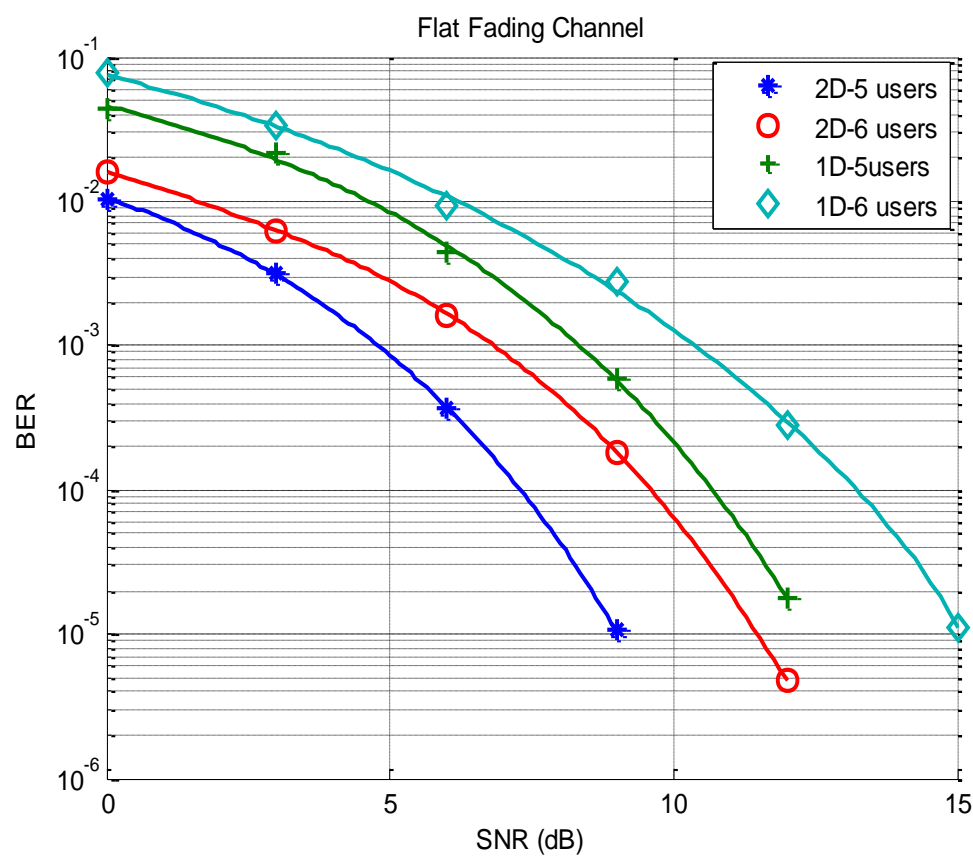

Figure (8): The performance of 2-D CDMA and 1-D CDMA in multi-path flat fading channel 


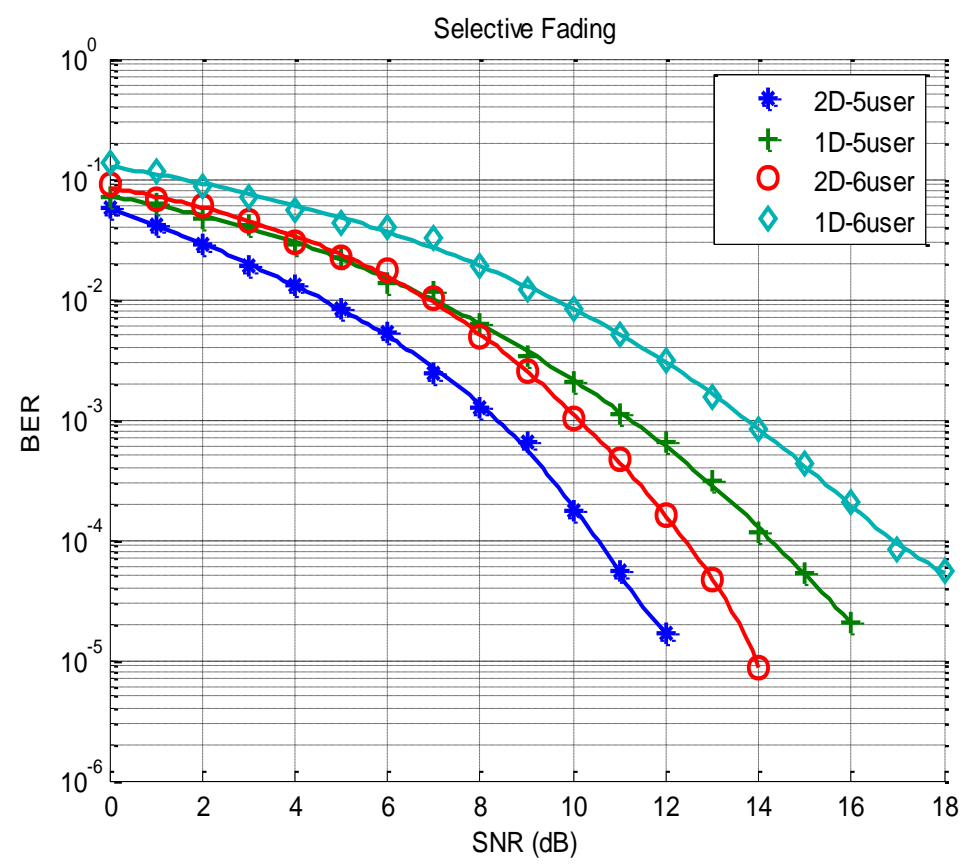

Figure (9): The performance of 2-D CDMA and 1-D CDMA in multi-path selective fading channel 


\section{تصميم ومحاكاة نظام تقسيم الرمز متعدد الوصول متوازي معتمد على تحويل هادامارد ثلاثي الابعاد}

$$
\text { علي طويج شاعدين }
$$

قسم الهندسة الكهربائية / كلية الهندسة / جامعة بغداد

تهدف انظمة الاتصالات المستقبلية الى زيادة معدلات ارسال البيانات و تحسين الكفاءة الطيفية و كذلك زيادة السعة.في هذه الدراسة تم اقتراح نظام تقسيم الرمز متعدد الوصول جديد ذو كفاءة طيفية عالية لتوليد وارسال رموز تقسيم

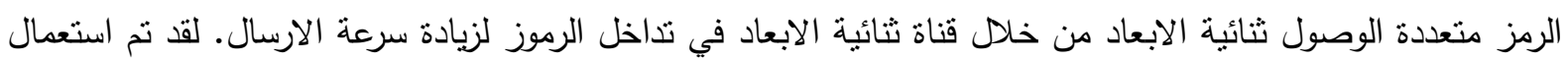

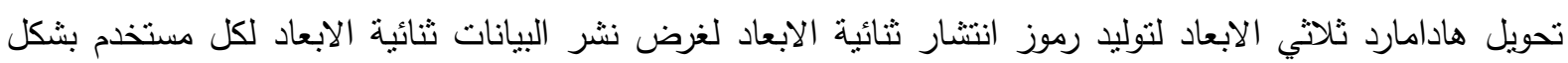

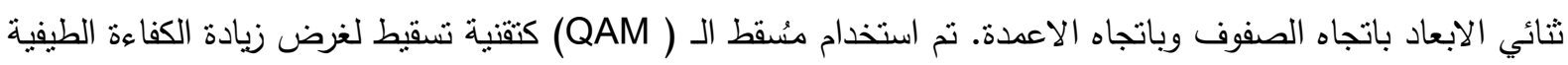

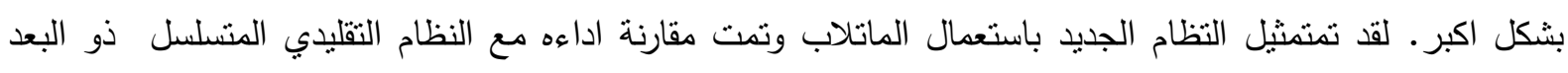

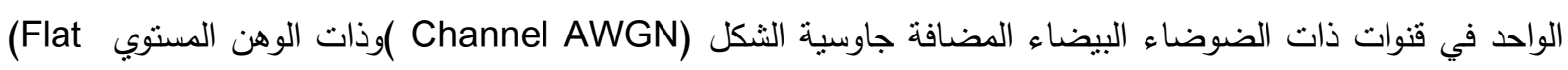

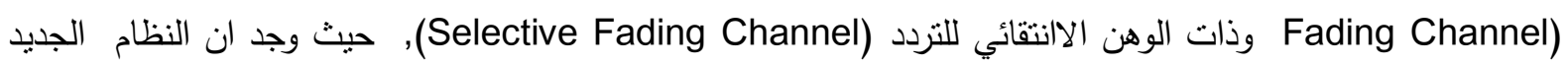
المنوازي افضل في الاداء واسرع من النظام التقليدي المنسلسل. 